\title{
The Optimization of Ridge Detection Algorithm for Multi-Component Signals, Entered into System in Time-Frequency Domain
}

\author{
Milad Daneshvar, Naser Parhizgar, and Homayoon Oraizi
}

\begin{abstract}
In this paper, we propose a method for applying time and frequency domain's representation to multicomponent signals. Our discussion is based on the method of ridge detection extraction taking into account the time and frequency domain by following the demodulation method, and the numerical results obtained by applying this method are evident compared to other methods that do not use demodulation. The simulation carried out on the examine signals indicate that the signal estimation can be accessed by initial estimation of the information carrier signals. In both noisy and noise-free environments, the frequency-time-based observation method is more accurate than the other methods.
\end{abstract}

Index Terms - Ridge Detection; Time Frequency Domain; Multi-Component Signal; Synchrosqueezing Transform

\section{INTRODUCTION}

There are many physical subsystems that produce synthetic signals, that usually integrate amplitude and frequency modulation waves. In many situations, optimality leads to the separation of multicomponent signals. As a result of the simplification of the above-mentioned signals over the past forty years, it can be mentioned in the case of Fourier, and half-wave transforms, and Fourier's integrals. Fast Fourier transform is part of the signal ridge detection that is included in the Fourier transform scheme [1]. This detection method is used to retrieve different components of the signal based on the signal ridge. It has been recently observed that the combination of local frequencies can lead to improvements in noise reduction. The foregoing method involves time-domain representation and acceptable approximation known as short time Fourier transform (STFT). However, in the basic formulas of this technique, it is not very useful to apply the method discussed for signals involving noise. Therefore, a factor called synchrosqueezing transform (SST) is used to understand the defect better. In the present discussion, we attempt to describe a specific modulation method based on the transform of second-order functions with the synchrosqueezing transform method to gain a better and more accurate representation of the signal

Published on October 24, 2019

Milad Daneshvar, Department of Electrical Engineering, Shiraz branch, Islamic Azad University, Shiraz, Iran.

(e-mail: milad_daneshvar@yahoo.com)

Nasser Parhizgar, Department of Electrical Engineering, Shiraz branch, Islamic Azad University, Shiraz, Iran.

(e-mail: author@lamar. colostate.edu)

Homayoon Oraizi, Iran University of Science and Technology (IUST), Tehran, Iran (e-mail: h_oraizi@iust.ac.ir). reconstruction results. The purpose of synchrosqueezing transform is to use a second-order estimation method called SST [4]. The benefits of using this method are to modify and optimize the Fourier transform accuracy so that its resolution is acceptable. The structure investigated in the current study discusses the Fourier transform called STFT [6], [7]. We try to focus on the operational structure of the signal ridge estimation and follow the displacement of the definition of the demodulation method by a structural algorithm.

\section{A. Initial definitions and notations}

We define the function $\mathrm{f}$ in the $\mathrm{L}^{1}(\mathrm{R})$ space, which is integrable and show the Fourier transform of the function with $\hat{f}$, and follows its normalization by the equation (1):

$$
\hat{f}(\eta)=\int_{\mathbb{R}} f(x) e^{-2 i \pi \eta x} d x
$$

The operator $\mathrm{g}$, the unstable derivatives of any order are defined by considering the STFT description of the function f:

$$
\mathrm{V}_{f}^{g}(\eta \cdot t)=\int_{\mathbb{R}} f(\tau) g^{*}(\tau-t) e^{-2 i \pi \eta(\tau-t)} d \tau
$$

The retrieval of the above function elements is defined as $\mathrm{f}_{\mathrm{k}}$ from a multi-component signals (MCS) function $\mathrm{f}$ :

$$
f(t)=\sum_{k=1}^{K} A_{k}(t) e^{-2 i \pi \emptyset_{k}(t)}=\sum_{k=1}^{K} f_{k}(t)
$$

In the above statement, $A_{k}(t)>0$ and $\emptyset_{k}^{\prime}(t)>0$ the signal Fourier transform of function $\mathrm{f}$ is calculated as follows:

$$
T I_{f}(\omega . t)=\sum_{k=1}^{K} A_{k}(t) \delta\left(\omega-\emptyset_{k}{ }^{\prime}(t)\right)
$$

The purpose of applying the FSST is to obtain the ideal Fourier transform [6-7], which estimates the time domain as follows:

$$
\begin{array}{r}
\widehat{\omega}_{f}(\eta \cdot t)=\frac{1}{2 \pi} \partial_{t} \arg V_{f}^{g}(\eta \cdot t) \\
=\eta-I_{m}\left\{\frac{1}{2 \pi} \frac{V_{f}^{g^{\prime}}(\eta \cdot t)}{V_{f}^{g}(\eta \cdot t)}\right\}
\end{array}
$$

In the above relation, $I_{m}\{\mathrm{x}\}$ represents the imaginary part of the complex number x. One of the most important features of FSST is that it can reconstruct the complex constants $V_{f}^{g}(\eta \cdot t)$, while applying the method in $(\eta \cdot t) \rightarrow$ 
$\left(\widehat{\omega}_{f}(\eta \cdot t) \cdot t\right)$. Basically, the components known as FSST follow the information about Fourier transforms and create the application of different modes of multi-component signal correction [6], [8]. The so-called synchrosqueezing operator is defined by the excitation edge $\gamma>0$, and the accuracy value $\lambda>0$ by applying an $\rho \in D(\mathbb{R})$ function. The defined space for functions having a condition of $\int_{R} \rho(x) d_{x}=1$ can be obtained with an acceptable approximation in the FSST calculation, by applying the following condition [6]:

$$
=\frac{1}{g(0)} \int_{\left\{\eta \cdot V_{f}(\eta \cdot t) \mid>\gamma\right\}} V_{f}^{g}(\eta \cdot t) \frac{1}{\lambda} \rho\left(\frac{\omega-\widehat{\omega_{f}}(\eta \cdot t)}{\lambda}\right) d_{\eta}
$$

Considering the condition $f \in \beta_{\Delta \cdot \varepsilon} . \tilde{\varepsilon}^{3}=\varepsilon$ and that $\mathrm{g}$ is the window coefficient, and its conjugate is also defined in the interval $[-\Delta . \Delta]$, where $\varepsilon$ be small enough, one can conclude:

$$
\begin{aligned}
& \left|V_{f}(\eta . t)\right|>\tilde{\varepsilon} \text { only if there exists } k \\
& \quad \in\{1 \ldots . . K\} \text { s.t. }(\eta . t) \in Z_{k}: \\
& \quad=\left\{(\eta . t) . \text { s.t. }\left|\eta-\emptyset_{k}^{\prime}(t)\right|<\Delta\right\}
\end{aligned}
$$

For all $\mathrm{k}$ coefficients and conditional pairs, we can conclude:

$$
k \in\{1 . \ldots . K\} .(\eta . t) \in Z_{k} . \text { s.t. }\left|V_{f}(\eta . t)\right|>\tilde{\varepsilon}
$$

And for the interval $\mathrm{k}$, we consider the constantcoefficient $\mathrm{C}$, over the whole range of real numbers [6].

\section{THE SECOND-ORDER SYNCHROSQUEEZING}

One of the limitations of FSST is the lack of conditions for the reconstruction of frequency modulation. The most recent method for discussing modulation is called via second-order synchrosqueezing transform (VSST) [8], [11]. The recent method defines phase states in the Synchrosqueezing operator with a second-order approximation. The VSST is derived from the new hybrid approximation of the second-order derivative of the function $\mathrm{f}$, which is followed by its final formula.

$$
\widetilde{q_{f}}(\eta \cdot t)=\frac{\partial_{t}\left(\partial_{t} V_{f}^{g}(\eta \cdot t) / V_{f}^{g}(\eta \cdot t)\right.}{2 i \pi-\partial_{t}\left(\partial_{t} V_{f}^{g}(\eta \cdot t) / V_{f}^{g}(\eta \cdot t)\right.}
$$

The above equation can be calculated through five different STFT components:

$$
\begin{gathered}
\widetilde{q_{f}}(\eta \cdot t) \\
=\frac{1}{2 i \pi} \frac{V_{f}^{g^{\prime \prime}}(\eta \cdot t) V_{f}^{g}(\eta \cdot t)-\left(V_{f}^{g^{\prime}}(\eta \cdot t)\right)^{2}}{V_{f}^{t g}(\eta \cdot t) V_{f}^{g^{\prime}}(\eta \cdot t)-V_{f}^{t g^{\prime}}(\eta \cdot t) V_{f}^{g}(\eta \cdot t)} \\
\text { By defining } \widetilde{\omega_{f}}(\eta \cdot t)=\frac{\partial_{\eta} V_{f}^{g}(\eta \cdot t)}{2 i \pi V_{f}^{g}(\eta \cdot t)} \text { and } \\
\widetilde{t_{f}}(\eta \cdot t)=t-\frac{\partial_{\eta} V_{f}^{g}(\eta \cdot t)}{2 i \pi V_{f}^{g}(\eta \cdot t)-V_{f}^{t g^{\prime}}(\eta \cdot t) V_{f}^{g}(\eta \cdot t)} \text { Definitions with a }
\end{gathered}
$$

new approximation in the function $\mathrm{f}$, it can be calculated as follows [11]:

$$
=\left\{\begin{array}{c}
\widehat{\omega}_{f}^{(2)}(\eta \cdot t) \\
\left.\widetilde{\mathcal{q}}_{f}(\eta \cdot t)\left(t-\tilde{\omega}_{f}(\eta \cdot t)\right)\right\} \text { if } \partial_{t} \tilde{t}_{f}(\eta \cdot t) \neq 0 \\
\widetilde{\omega}_{f}(\eta \cdot t) \quad \text { otherwise }
\end{array}\right.
$$

Which in the latter relation $\mathcal{R}_{e}\{X\}$ represents the real part of $X$, and this cannot be kept away from $\widetilde{\omega}_{f}(\eta \cdot t)=\mathcal{R}_{e}\left\{\widetilde{\omega}_{f}(\eta \cdot t)\right\}$, and can be deduced if Gaussian coefficient of reinforcement, and $f(t)=A(t) e^{2 i \pi \emptyset(t)}$ $: \widehat{\omega}_{f}^{(2)}(\eta \cdot t)=\emptyset^{\prime}(t)$

\section{A. STFT calculation and Synchrosqueezing transform}

In many operational calculations, the signal $\mathrm{f}$ has a finite signal length that is defined in the closed range $[0, \mathrm{~T}]$ and its normalization are achieved by: $f\left(\frac{n T}{N}\right)_{n=0 \ldots \ldots N-1}$. On the topic of the discussion of this article, $\mathrm{N}$ is displayed up to value 2 and according to the calculations of $\mathrm{g}$ in the interval $\left[-\frac{L T}{N} \cdot \frac{L T}{N}\right]$ where $L<N / 2$ is given by Obtain the above STFT calculations as follows:

$$
\mathrm{V}_{f}^{g}(\eta . t)
$$

$$
\begin{aligned}
& =\int_{\mathbb{R}} f(t+\tau) g(\tau) e^{-2 i \pi \eta(\tau-t)} d \tau \\
& =\int_{-\frac{L T}{N}}^{\frac{L T}{N}} f(t+\tau) g(\tau) e^{-2 i \pi \eta \tau} d \tau \\
& \approx \frac{T}{N} \sum_{n=-L}^{L} f\left(t+\frac{n T}{N}\right) g\left(\frac{n T}{N}\right) e^{-i 2 \pi \frac{n T}{N} \eta}
\end{aligned}
$$

And in the computational approximation mode, the following formula can be accessed:

$$
\begin{gathered}
\mathrm{V}_{f}^{g}\left(\frac{p}{T} \cdot \frac{q T}{N}\right) \approx \\
\frac{T}{N} \sum_{n=-L}^{L} f\left(\frac{(q+n) T}{N}\right) g\left(\frac{n T}{N}\right) e^{-i 2 \pi \frac{n p}{N}}= \\
\frac{T e^{2 i \pi \frac{p L}{N}}}{N} \sum_{n=0}^{2 L} f\left(\frac{(q+(n-L)) T}{N}\right) g\left(\frac{(n-L) T}{N}\right) e^{-i 2 \pi \frac{n p}{N}}:= \\
e^{2 i \pi \frac{n p L}{N}} \sum_{n=0}^{2 L} S(q \cdot n) e^{-i 2 \pi \frac{n p}{N}}
\end{gathered}
$$

In the last calculation, the FFT coefficient can also be obtained. In the usual case, the value of each $\mathrm{q}$ is given by order of $(S(q \cdot n))_{n}$.

In the order of size $N_{f}>N$ by adding the number of $N_{f}-$ $N$ to the given function; the above approximation can be calculated as follows:

$$
\begin{gathered}
\mathrm{V}_{f}^{g}\left(\frac{N}{N_{f}} \frac{p}{T} \cdot \frac{q T}{N}\right) \approx \\
\frac{T}{N} \sum_{n=-L}^{L} f\left(\frac{(q+n) T}{N}\right) g\left(\frac{n T}{N}\right) e^{-i 2 \pi \frac{n p}{N}}= \\
\frac{T e^{2 i \pi \frac{p L}{N}}}{N} \sum_{n=0}^{2 L} f\left(\frac{(q+(n-L)) T}{N}\right) g\left(\frac{(n-L) T}{N}\right) e^{-i 2 \pi \frac{n p}{N}}:= \\
e^{2 i \pi \frac{n p L}{N}} \sum_{n=0}^{2 L} S(q \cdot n) e^{-i 2 \pi \frac{n p}{N}}
\end{gathered}
$$

Since $\mathbf{V}_{f}^{g}\left(\frac{N}{N_{f}} \frac{p}{T} \cdot \frac{q T}{N}\right)$ is approximated by an FFT coefficient, the first - half cycle of the whole signal period is then calculated such that $\mathbf{V}_{\boldsymbol{f}}^{\boldsymbol{g}}$ in the Fourier transform network is approximated.

$$
\left\{0, \frac{T}{N} \ldots . . \frac{(N-1) T}{N}\right\}><\left\{0 . \frac{N}{N_{f}} \frac{1}{T} \ldots . \frac{N}{N_{f}} \frac{N_{f} / 2-1}{T}\right\}
$$




\section{B. Approximation of signal ridges}

Any replacement method based on synchrosqueezing requires approximate methods of course in $\left(\boldsymbol{t} . \emptyset_{k}^{\prime}(\boldsymbol{t})\right)$ interval where the transform depends on the application of spectral-based methods capable of accessing Fourier transform types. According to the recent discussion, we are trying to achieve a classic definition of ridge detection. The benefits and influence of parameter differences in the accuracy of the approximation and non-noise ability of the components are controversial.

\section{PROPOSED RIDGE EXTRACTION ALGORITHM}

The approximate calculation of the ridge detection in the interval $\left(\boldsymbol{t} . \emptyset_{\boldsymbol{k}}^{\prime}(\boldsymbol{t})\right)$ returns to the knowledge of numerical computation for $\mathrm{k}$ states. We can define an algorithm similar to that described in [5] with the aim of computing minimum finite functions using the following formula [13].

$$
\begin{aligned}
& E_{f}\left(\psi_{1} \ldots . \psi_{k}\right)=\sum_{k=1}^{k}-\int_{\mathbb{R}}\left|\mathrm{T} F_{f}\left(\psi_{k} \quad(t), t\right)\right|^{2} d_{t} \\
& +\int_{\mathbb{R}} \lambda \psi_{k}^{\prime}(t)^{2} d_{t}+\beta \psi_{k}^{\prime \prime}(t)^{2} d_{t}
\end{aligned}
$$

In the aforementioned equation $\mathrm{T}_{f}$ is one of the Laplace transform coefficients calculated by $\mathrm{T}_{f}^{g}$ and $\mathrm{V}_{f}^{g}$ or $\mathrm{TV}_{f}^{g}$. However, as observed, the earlier equation cannot be a definitive factor for calculating the edge detection coefficient.

\section{A. Influence of zero-padding on the signal ridge approximation}

First, let's discuss the effect of zero-padding on the ridge approximation. We note that for each case a monocomponent signal can be used to approximate the term $\emptyset_{k}^{\prime}\left(t_{n}\right)$ such that:

$$
\Psi\left(t_{n}\right)={ }_{\eta}^{\operatorname{argmax}}\left|\mathrm{V}_{f}^{g}\left(\eta \cdot t_{n}\right)\right|^{2}
$$

As noted in [14], for the noisy state of the signal $f(t)=A(t) e^{2 i \pi \phi(t)}$, for example, we can mention $\tilde{f}\left(t_{n}\right)=f\left(t_{n}\right)+\epsilon\left(t_{n}\right)$ that in the recent relation, $\epsilon$ represents the effect of white noise whose coefficient of variation is $\sigma_{\epsilon}^{2}$. Of course, the choice of coefficient of variation can be followed from the below relation.

$$
\Delta \Psi\left(t_{n}\right):=\Psi\left(t_{n}\right)-\emptyset^{\prime}\left(t_{n}\right)
$$

The parameter $\mathrm{g}$ is called the Gaussian window coefficient, which can be calculated by the following equation.

$$
g(x)=\frac{1}{\sqrt{2 \pi} \sigma} e^{-\frac{x^{2}}{2 \sigma^{2}}}
$$

According to the calculations, it can be proved that [14]:

$$
\begin{gathered}
\operatorname{Bias}\left\{\Delta \Psi\left(t_{n}\right)\right\} \sim N \rightarrow+\infty \sum_{k=1}^{+\infty} \frac{2 \pi \emptyset^{(2 k+1)}\left(t_{n}\right) \sigma^{2 k}}{2^{k K !}} \\
\operatorname{Var}\left\{\Delta \Psi\left(t_{n}\right)\right\} \sim N \rightarrow+\infty \frac{\sigma_{\epsilon}^{2}}{8 \sqrt{\pi}\left|\mathrm{A}\left(t_{n}\right)\right|^{2}} \cdots \\
{\left[1+\frac{\sigma_{\epsilon}^{2} T}{2 N \sqrt{\pi} \sigma\left|\mathrm{A}\left(t_{n}\right)\right|^{2}}\right] \frac{T}{N \sigma^{3}}}
\end{gathered}
$$

The above results are important. Therefore, these calculations include the real coefficient that $\mathrm{V}_{f}^{g}\left(\eta \cdot t_{n}\right)$ is the only present frequency network available through FFT calculations. Previously for ridge detection dependent calculations, we studied and computed the continuous frequency signal with coefficients of $\lambda$ and $\beta$. It depicts a set of frequency bandwidths at the signal resolution level. Considering the results of the Fourier transform in the set of frequencies $\left(\frac{N}{N_{f}} \frac{p}{T}\right)_{p=0 \ldots . .} N_{f / 2-1}$, it is possible to investigate the approximation quality of the signal separation. In the case of frequency instability, a better understanding of ridge detection can be accessed. We're not just looking at the effect of Zero-Padding, but also on the level of noise. Since the study of linear functions is constrained, we will analyze three different signal types, which will include STFT state representation. These modes are shown in Figure 1. The results shown in 3D figure 1 indicate that the noise-free signal in the linear state using STFT and VSST in the ridge detection using MSE operators will be ones, although this property depends on the fact that the optimal coefficients signal adjustment should be at the maximum STFT value with VSST. For other types of signals, the detection will be based on the STFT behaviors with a bit change over the VSST. More accurately since the FSST is calculated assuming the low-precision approximation of the detection frequency, the results obtained for the ridge detection estimation parameters will be more precise and accurate than the calculations in the Fourier transform detection. For the reasons given, we can focus on the signals shown in Figures $\mathrm{A}$ and $\mathrm{B}$ based on the MSE detection error. Therefore, we will not be much interested in looking at part $\mathrm{C}$ of Figure 1. Since signal modulation is of particular importance, thus, the method discussed in low-frequency signals has no opportunity for study and discussion. The result of the recent study is that frequency resolution for the purpose of so-called ridge estimation occurs without any dependence on signal modulation. One of the modulation requirements of small type signals is access to frequency resolution.
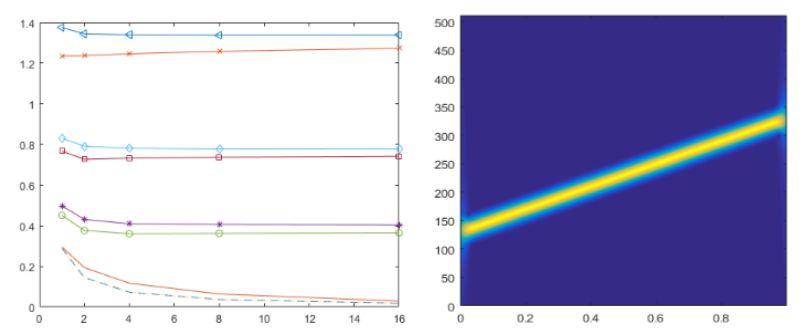

Figure A,D
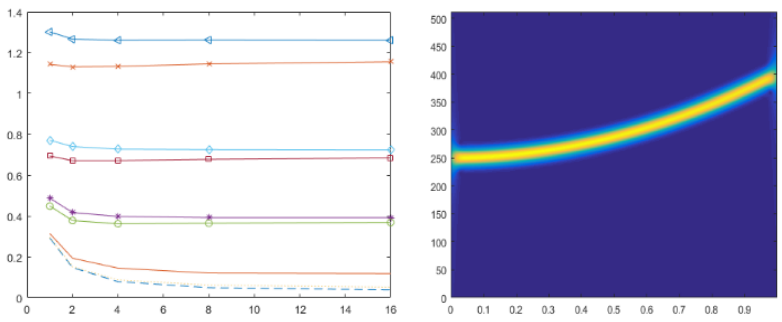

Figure B,E 


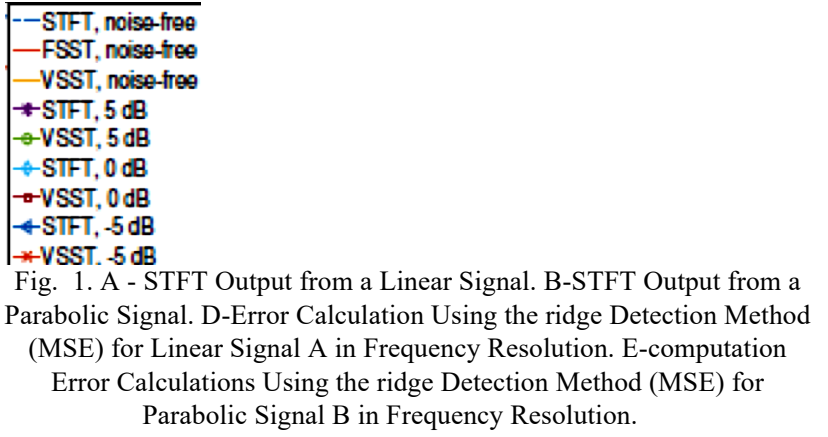

We strive to gain the necessary understanding of the available conditions for noisy ambient signals, so we follow the ridge detection effects on linear, parabolic, and sinusoidal signals with an SNR ratio of six dB. The results of part D from Figure 1 are shown for the last period of the studied signal period. It can be clearly deduced from parts D and $\mathrm{E}$ from Figure 1 that the higher the frequency resolution the finer the magnitude of the $\mathrm{Nf}$ coefficient, and this feature can lead to greater signal approximation accuracy. In addition, the approximation quality created by ridge detection in VSST deployment modes is much more useful than STFT deployment. Ridge detectors are less sensitive to noise in this case. Finally, we note that it is appropriate to simulate $\quad \mathrm{N}_{\mathrm{f}}=8 \mathrm{~N}$ for the simulation since it can achieve the ridge detection objectives.

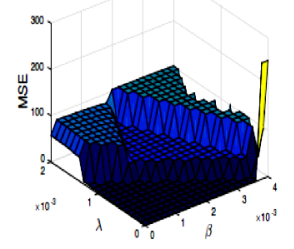

A

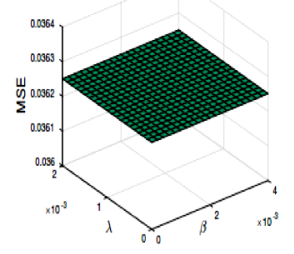

D

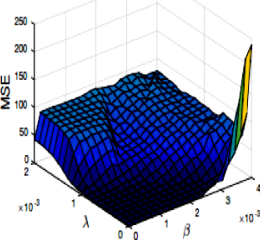

B

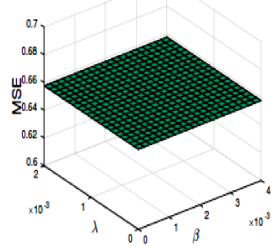

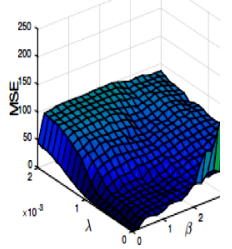

C

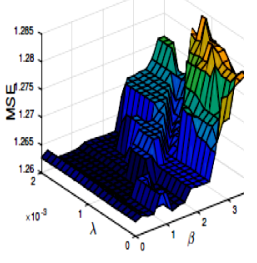

F
Fig. 2. A-MSE shows the so-called ridge estimation for the linear function of part A of Figure 1, taking $V_{f}^{g}$ as the Fourier transform with the noise factor $\mathrm{Nf}=8 \mathrm{~N}$. B - This figure is similar to part $\mathrm{A}$, but has been examined at zero decibels. $\mathrm{C}$ - This figure is similar to part $\mathrm{A}$, but has been examined at the six $\mathrm{dB}$ level. D-MSE depends on the so-called ridge estimation for the linear function of part A of Figure 1 considering $T V_{f}^{g}$ as a representation of the Fourier transform. E - This figure is similar to part D but has been examined at zero decibels. $\mathrm{H}$ - This figure is similar to part D but has been examined at the six dB level.

\section{B. Demodulation operator definition}

By defining the ridge estimation we can specify the demodulation algorithm for a mono-component signal $f(t)=A(t) e^{2 i \pi \emptyset(t)}$. For any calculation and estimation of the defined function, we can compute the estimate $\psi(t)$. For linear modes such as $\emptyset(t)=a t+b t^{2}$, and $\psi(t)$ is an approximation of $a+2 b t$. Therefore to calculate the function $\mathrm{f}(\mathrm{t})$ by $e^{-2 i \pi(\phi(t) t / 2)} \quad$ and if the $\operatorname{IMF}$ (Intrinsic Mode
Functions) estimate is accurately calculated. It is recommended to obtain a $f_{D}$ demodulation signal with a constant frequency of $\mathrm{a} / 2$. Therefore, it is important to pay attention to the demodulation method to access the signal linearization properties. Especially in the context of decreasing the order of second-order and higher signals. Thus, performing the demodulation function $f(t)=$ $A(t) e^{2 i \pi \varnothing(t)}$ with the operator $e^{-2 i \pi\left(\int_{0}^{t} \psi(x) d_{x}-\psi_{0} t\right)}$. That parameter $\psi_{0}$ it represents a constant positive frequency is fully applicable:

$$
f_{D}(t)=f(t) e^{-2 i \pi\left(\int_{0}^{t} \psi(x) d_{x}-\psi_{0} t\right)}
$$

As shown in Figure 3, the parameter $\psi_{0}$ is $95 \mathrm{kHz}$ for the three different states. In Figure 3, we illustrate the VSST of noise-free modes.
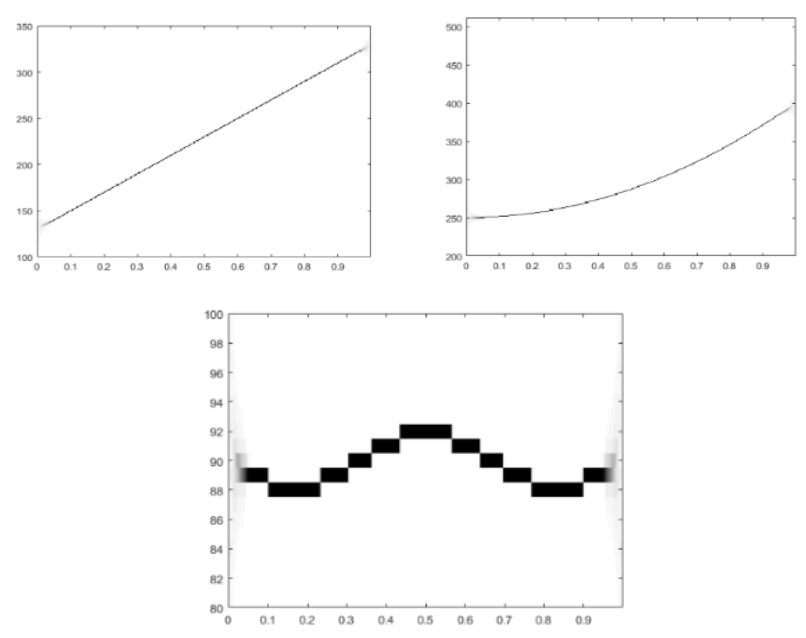

figures A, B, C from right to left
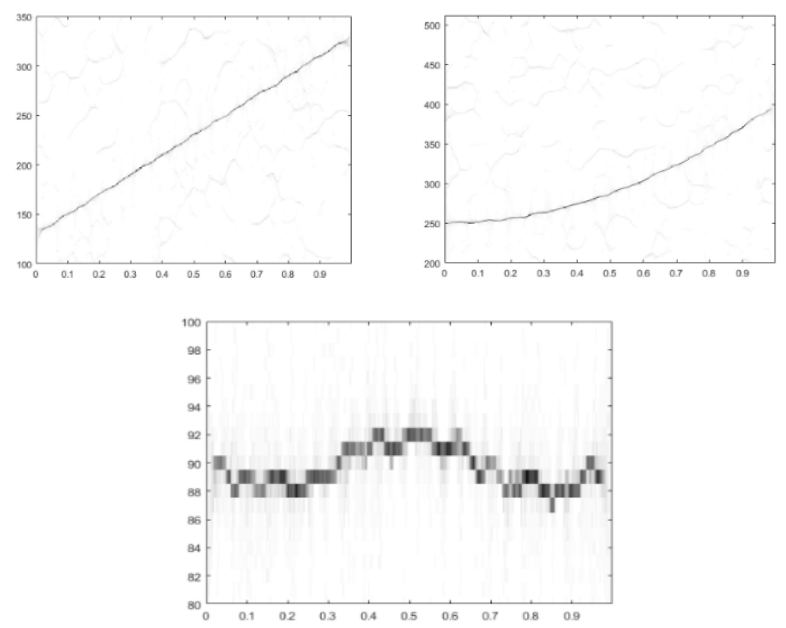

figures D, E, F from right to left
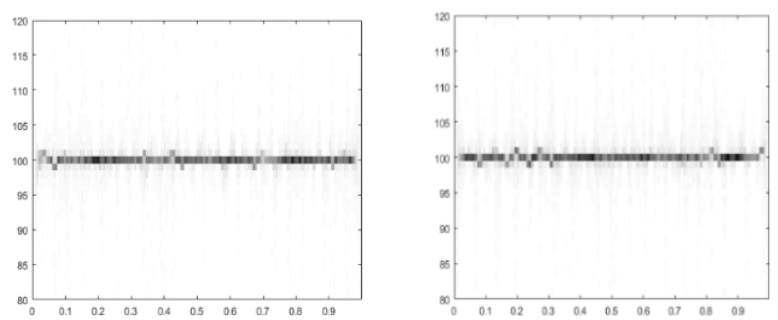


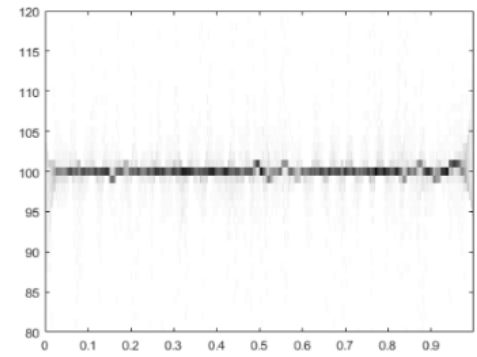

figures $\mathrm{G}, \mathrm{H}$, I from right to left

Fig. 3. A-VSST from a noise-free linear function. B-VSST from the noise-free parabolic function. C-VSST from the sinusoidal function. DVSST from a linear function with zero dB noise level. E-VSST from the parabolic function with zero dB noise level. F-VSST from a sinusoidal function with zero $\mathrm{dB}$ noise level. G-signal level demodulated from the original D signal. H-signal demodulated from main signal E. I- signal demodulated from a main signal $\mathrm{F}$.

By visualizing the VSST caused by the demodulated signals in three modes, we obtain the aforesaid results with $\mathrm{N}_{\mathrm{f}}=8 \mathrm{~N}$. In spite of high noise environments, the above method can also be applied well in noisy environments. Now let's look at how the above method works when dealing with multi-component signals. We show three different modes in Figure 4. By applying the second method algorithm to compute VSST with $\mathrm{N}_{\mathrm{f}}=8 \mathrm{~N}$, one can estimate the functions for access to $\psi_{1}, \psi_{2}$ and $\psi_{3}$ In the order that all three demodulation signals were performed.

$$
f_{D . k(t)}=f(t) e^{-2 i \pi\left(\int_{0}^{t} \psi(x) d_{x}-\psi_{0} t\right)} \quad . k=1.2 .3
$$

The VSST coefficient of the three signals $\left(f_{D . k}\right)_{k}=1.2 .3$ is shown in sections B and D of Figure 4, where the SNR in the original signal is zero $\mathrm{dB}$. It should be noted that the parameter $f_{D . k}$ represents the $\mathrm{K}_{\text {th }}$ state of the demodulated signals.
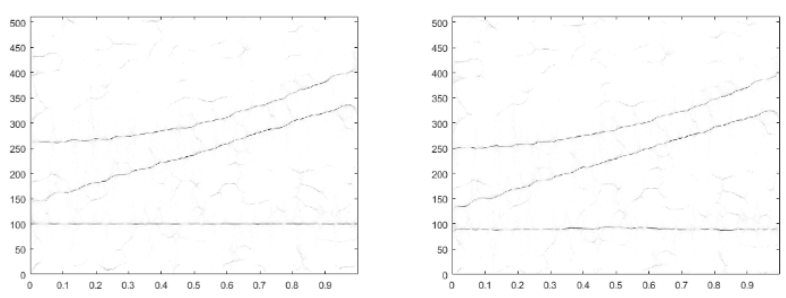

figures A,B from right to left
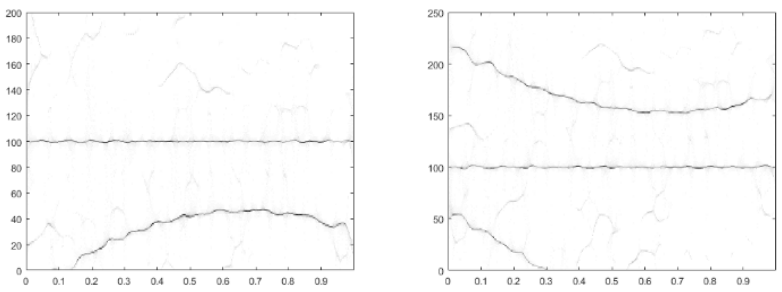

figures C,D from right to left

Fig. 4. A-Three-mode zero-dB signal level B-VSST fD,1. C - VSST fD,2 . D - VSST fD,3

\section{Determine the window length}

The optimal calculation of the window length parameter can be obtained by considering the Gaussian coefficient $g$ eg, $\quad(x)=\frac{1}{\sqrt{2 \pi \sigma}} e^{-\frac{x^{2}}{2 \sigma^{2}}}$, where the parameter length discussed is controlled by the sigma parameter can be calculated. We try to calculate the above parameter value in a more centralized way. We follow the application of the focusing parameter in the calculation of the parameter by using a VSST called Shannon entropy [19], [20]
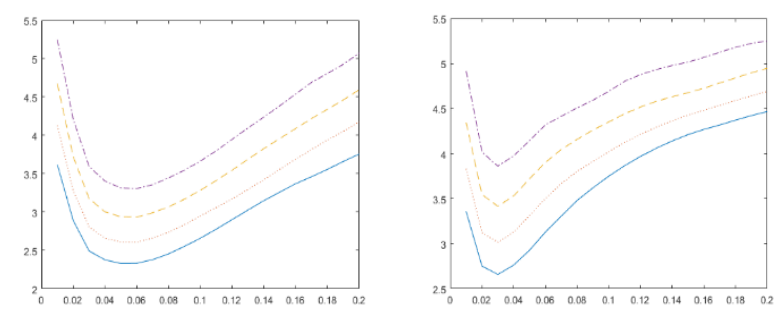

$$
\begin{aligned}
-N_{f} & =N \\
-N_{f} & =2 N \\
-N_{f} & =4 N \\
-N_{f} & =8 N
\end{aligned}
$$

Fig. 5. A - Representation of the Rényi entropy effect with respect to VSST of Figure 4 part A, of course, Noise mode with respect to the sigma parameter with respect to the signal resolution at different frequencies. The SNR is five $\mathrm{dB}$.

$=-\sum_{p=0}^{\frac{N_{f}}{2-1}} \sum_{n=0}^{N-1} \frac{\left|T V_{f}^{g}\left(\frac{N}{N_{f}} \frac{p}{T} \cdot t_{n}\right)\right|}{\left\|T V_{f}^{g}\right\|_{1}} \log _{2} \frac{\left|T V_{f}^{g}\left(\frac{N}{N_{f}} \frac{p}{T} \cdot t_{n}\right)\right|}{\left\|T V_{f}^{g}\right\|_{1}}$

Or via Rényi entropy:

$$
=\frac{1}{1-\alpha} \log _{2}\left(\left(\sum_{p=0}^{\frac{N_{f}}{2-1}} \sum_{n=0}^{N-1} \frac{\left|T V_{f}^{g}\left(\frac{N}{N_{f}} \frac{p}{T} \cdot t_{n}\right)\right|}{\left\|T V_{f}^{g}\right\|_{1}}\right)^{\alpha}\right)
$$

The operation output reports, of course, are very similar. Note to the phrase:

$$
\left\|T V_{f}^{g}\right\|_{1}=\sum_{p=0}^{\frac{N_{f}}{2-1}} \sum_{n=0}^{N-1}\left|T V_{f}^{g}\left(\frac{N}{N_{f}} \frac{p}{T} \cdot t_{n}\right)\right|
$$

It is very important to decide which of these methods, but note that they are useful for low noise levels [19]. A brief look at the illustrations in Figure 5 shows that Rényi entropy can be extracted with an alpha value of 3 from the VSST signal shown $\mathrm{f}$ in figure 4 in part $\mathrm{A}$. Another point to note is that the values presented for sigma are used at noise levels below zero decibels, although the values in this text are constant values for each case. Note that in each case, the result is independent of frequency. Here we note that Rényi entropy is calculated through second-order computational

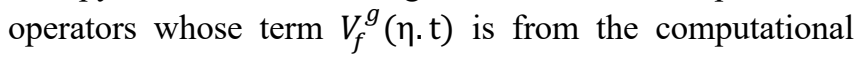
interval $\left|R_{e}\left\{V_{f}^{g}(\eta \cdot \mathrm{t})\right\}\right|>\gamma_{1}\left|I_{m}\left\{V_{f}^{g}(\eta \cdot \mathrm{t})\right\}\right|>\gamma_{2}$ and, of course, the parameters $\gamma_{1}$ and $\gamma_{2}$ follow the standard division of $R_{e}\left\{V_{f}^{g}(\eta \cdot \mathrm{t})\right\}$ (resp. $\left.I_{m}\left\{V_{f}^{g}(\eta \cdot \mathrm{t})\right\}\right)$. The choice for the threshold to the current standard occurs with the STFT coefficient based on the white noise level of the Gaussian coefficient calculation process. Therefore, methods based on Rényi entropy are developed to determine the sigma coefficient to estimate the tolerance of noisy 
environments. According to part $\mathrm{D}$ of Figure 5, it is impossible to calculate the $\mathrm{k}$ state of the third algorithm alone. However, according to the ridge detection method, it can be said that the computation of the sigma coefficient depends on the maximum state calculated for $\mathrm{k}$. The best way to calculate the above is to use the following equation:

$$
E_{R}(\sigma)=\frac{\sum_{k=0}^{k} \sum_{n=0}^{N-1}\left|T V_{f}^{g}\left(\psi_{k}\left(t_{n}\right) \cdot t_{n}\right)\right|^{2}}{\sum_{p=0}^{\frac{N_{f}}{2-1}} \sum_{n=0}^{N-1}\left|T V_{f}^{g}\left(\frac{N}{N_{f}} \frac{p}{T} \cdot t_{n}\right)\right|^{2}}
$$

In the above relation, $E_{R}$ represents the energy present in any ridge detection, but we must remember that the sigma coefficient is independent of the Gaussian coefficient $\mathrm{g}$. In fact, $E_{R}$ depends on part of the energy set present in ridge detection. We assume that $E_{R}(\sigma)$ is applicable for the same signal and different noise levels and for $N_{f}=N$ as shown in Figure 6 . Recall that the numerical value initially is close to the value calculated by Rényi entropy for noise levels below zero $\mathrm{dB}$ and that the information obtained by the ridge detection method at lower noise levels is magnified. In fact, what is of particular interest in the Rényi entropy method is the finding of the sigma variable at the noise level of $5 \mathrm{~dB}$. As with other methods, it is possible to determine the sigma coefficient in highly noisy environments. Finally, paying attention to similar results for different values of the $\mathrm{N}_{\mathrm{f}}$ coefficient can be very important.
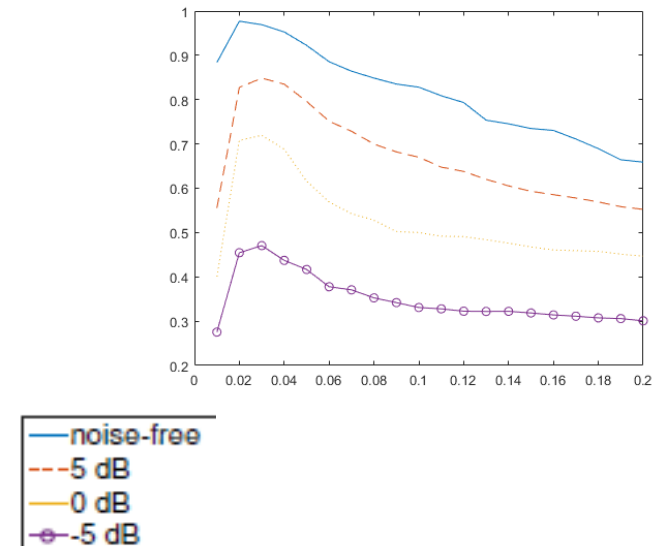

Fig. 6. ER calculation depending on the partial of energy stored in the VSST calculated at the ridge detection using the sigma coefficient and considering the three noisy modes, $5 \mathrm{~dB}$ noise level, and $5 \mathrm{~dB}$ noise level

\section{Signal Reconstruction Method: Including Noise-free Ambient Mode}

In this part, we try to pursue the quality of reconstruction modes in noise-free mode with parameter selection. The phenomenon known as the Fourier transform window is Gaussian, and its length can be defined according to what has been discussed in the previous sections. Therefore, ridge detection and signal reconstruction mode are defined in the case of applying a small value for $\gamma$. For example, consider the value $\gamma=10^{-3}$, such that all non-zero constants for each case are chosen according to the signal studied. Since the calculation of the signal ridge detection directly affects the frequency resolution, therefore, it is important to investigate the effect of $\mathrm{N}_{\mathrm{f}}$ on the ridge detection calculations. Furthermore, showing what the results of the demodulation application of the Fourier transform accesses are considered is clearly related to the VSST and the role of the parameter d.

\section{E. Signal Reconstruction Method: Including Noise Ambient Mode}

In this part, we test the effect of noise on the sensitivity of the method discussed for the recovery mode; of course it is consisting of two types of displayed signals. According to what was studied in noise-free mode, the signal ridge computation at $\mathrm{N}_{\mathrm{f}}=8 \mathrm{~N}$ has better reconstruction results, so we use the above value in the simulations. Again, by using the Gaussian window with the calculated sigma length as before, we choose the signal threshold value as $\gamma=10^{-3}$. The results depicted in the primary section of Figure 9. To show the SNR output in the reconstruction mode, when the coefficient $d$ is set to zero or five, similar to that applied to the first and second modes by applying the SNR coefficient. It was shown. The difference in terms of signal regeneration characteristic between the two methods is increasing, however, when the noise level is reduced, the benefit of the first demodulation is not significant as the VSST is optimized for linear characteristics. Moving to the study of the proposed signal further clarifies the benefits of applying demodulation methods, therefore, when the state is very different for the once situation, the amount of optimization generated in the signal will be much clearer. Finally, we try to pay more attention to the optimization method, when using the parameter of noise-free environment d plays a key role in the creation of quality demodulation, so that is why we will focus specifically on the above parameter.

\section{CONCLUSION}

In this study, we attempted to define a new algorithm for existing options for detecting multi-component signals. Therefore, this study was performed considering the frequency-time domain. The basis of the proposed methods could lead to ridge signal detection, which is considered as a demodulation detection method. By using a frequency with a clear resolution, a comparison was made between the frequency discontinuities with respect to FFT, which helped to calculate and display the Fourier transform components. The simulations performed on the test signals show that the initial estimation of the information carrier signals can be accessed by signal demodulation. In both noise-free and noisy environments, the frequency-time-observation-based approach is more accurate than the other methods. Simulations performed on real; rather than laboratory, signals indicate that the number of states can be defined according to the time and the constraints of the variable signal reconstruction, however, observing the constraints of the signal reconstruction is one of the things that can give us the background for further research in the field that will show in the near future. 


\section{REFERENCES}

[1] I. Daubechies, J. Lu, and H-T. Wu, "Synchrosqueezed wavelet transforms: an empirical mode decomposition-like tool," Applied and Computational Harmonic Anal-ysis, vol. 30, no. 2, pp. 243-261, 2011.

[2] G. Thakur, E. Brevdo, N.S. Fuckar," and H-T. Wu, "The synchrosqueezing algorithm for time-varying spectral analysis: robustness properties and new paleoclimate applications," Signal Processing, 2012.

[3] C. Franco, P-Y. Gumery,' N. Vuillerme, A. Fleury, and J. FontecaveJallon, "Synchrosqueezing to investigate cardio-respiratory interactions within simulated volu-metric signals," in Proceedings of the 20th European Signal Processing Conference (EUSIPCO). IEEE, 2012, 939-943.

[4] D. Iatsenko, A. Bernjak, T. Stankovski, Y. Shiogai, P.J. Owen-Lynch, P.B.M. Clarkson, P.V.E. McClintock, and A. Stefanovska, "Evolution of cardiorespiratory interac-tions with age," Philosophical Transactions of the Royal Society A: Mathematical, Physical and Engineering Sci-ences, vol. 371, no. 1997, 2013.

[5] G. Thakur and H-T. Wu, "Synchrosqueezing-based recovery of instantaneous frequency from nonuniform samples," SIAM Journal on Mathematical Analysis, vol. 43, no. 5, pp. 2078-2095, 2011

[6] I. Daubechies, J. Lu, and H.-T. Wu, "Synchrosqueezed wavelet transforms: An empirical mode decomposition-like tool," Appl. Comput. Harmon. Anal., vol. 30, no. 2, pp. 243-261, 2011.

[7] T. Oberlin, S. Meignen, and V. Perrier, "The Fourier-based synchrosqueezing transform," in IEEE Int. Conf. Acoust., Speech., Sig. Proc. (ICASSP), 2014, pp. 315-319.

[8] H.-T. Wu, "Adaptive analysis of complex data sets," $\mathrm{PhD}$, Princeton University, 2012

[9] S. Wang, X. Chen, G. Cai, B. Chen, X. Li, and Z. He, "Matching demodulation transform and synchrosqueezing transform in timefrequency analysis,” IEEE Trans. Sig. Proc., vol. 62, no. 1, pp. 69-84, 2014.

[10] T. Oberlin, S. Meignen, and V. Perrier, "Second-order synchrosqueezing transform or invertible reassignment? Towards ideal time-frequency representations," IEEE Trans. Sig. Proc., vol. 63, no. 5, pp. 1335-1344, March 2015.

[11] D.-H. Pham and S. Meignen, "High-order synchrosqueezing transform for multicomponent signals analysiswith an application to gravitational-wave signal," IEEE Trans. Sig. Proc., vol. 65, no. 12, pp. 3168-3178, 2017.

[12] S. Meignen, D.-H. Pham, and S. McLaughlin, "On demodulation, ridge detection and synchrosqueezing for multicomponent signals," IEEE Trans. Sig. Proc., vol. 65, no. 8, pp. 2093-2103, 2017.

[13] H. Yang, "Statistical analysis of synchrosqueezed transforms," Appl. Comput. Harm. Anal., vol. doi.org/10.1016/j.acha.2017.01.001, 2017.

[14] J. Xiao and P. Flandrin, "Multitaper time-frequency reassignment for nonstationary spectrum estimation and chirp enhancement," IEEE Trans. Sig. Proc., vol. 55, no. 6, pp. 2851-2860, 2007.

[15] D. W. Griffin and S. L. Jae, "Signal estimation from modified shorttime Fcourier transform," IEEE Trans. Acoust., Speech, and Sig. Proc., vol. 32, no. 2, pp. 236-243, 1984.

[16] R. Behera, S. Meignen, and T. Oberlin, "Theoretical analysis of the second-order synchrosqueezing transform," Appl. Comput. Harm. Anal., vol. , doi:10.1016/j.acha.2016.11.001, 2016.

[17] G. Thakur and H.-T. Wu, "Synchrosqueezing-based recovery of instantaneous frequency from nonuniform samples.” SIAM J. Math. Anal., vol. 43, no. 5, pp. 2078-2095, 2011.

[18] S. Wang, X. Chen, G. Cai, B. Chen, X. Li, and Z. He, "Matching demodulation transform and synchrosqueezing in time-frequency analysis," Signal Processing, IEEE Transactions on, vol. 62, no. 1, pp. 69-84, 2014.

[19] movements using multicomponent am-fm decomposition," IEEE journal of biomedical and health informatics, vol. 19, no. 5, pp. 1672$1681,2015$.

[20] R. Sharma, L. Vignolo, G. Schlotthauer, M. A. Colominas, H. L. Rufiner, and S. Prasanna, "Empirical mode decomposition for adaptive am-fm analysis of speech: a review," Speech Communication, vol. 88, pp. 39-64, 2017.

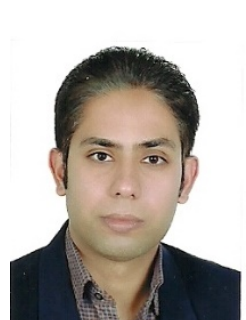

Milad Daneshvar was born in Shiraz, Iran on May 5, 1984. He received B.S. degree from the Shiraz University of Technology, Shiraz, Iran and M.S degree from the Boushehr Branch, Islamic Azad University, Boushehr, Iran and ph.D. Condidate from the Shiraz Branch, Islamic Azad university,Shiraz, Iran, all in Electrical Engineering, in 2007, 2013 and 2018 respectively. He has translated several textbooks in Farsi. He has authored over 40 papers in Electrical Engineering conferences in Iran. In 2016, he was as a reviewer at the 6th.world congress on electrical engineering, computer science and information technology, Barcelona, Spain and In 2015 at the 2nd. International Congress of Electrical Engineering,Tehran, Iran. His major research interest include Electromagnetic Engineering, Signal Processing.

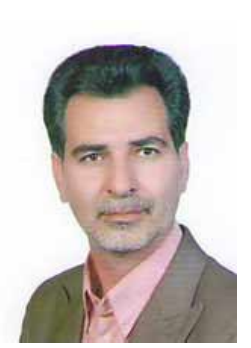

Naser Parhizgar was born in Shiraz, Iran on October 24, 1968. He received B.S. and M.S degrees from Shiraz University,Shiraz, Iran and ph.D. degree from the Iran University of Science and Technology(IUST), Tehran, Iran, all in Electrical Engineering, in 1992, 2007 and 2013 respectively. In 2010, he joined the Department of Electrical Engineering Shiraz Branch, Islamic Azad University, Shiraz, Iran. He was as a Distinguished researcher in Najafabad Branch, Islamic Azad University, Isfahan, Iran, 2007 and Fars Science and Research Branch, Islamic Azad University,Shiraz,Iran, 2011. His major research interest include Communication Engineering, Signal Processing, Aray Processing, RADAR

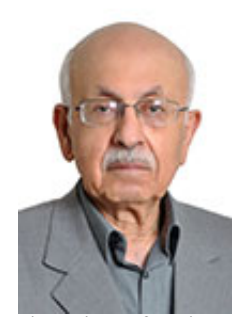

Homayoon Oraizi(SM) received B.E.E. degree from American University of Beirut, Lebanon, in 1967, M.Sc. and ph.D. degrees in electrical engineering from Syracuse University, Syracuse, NY, in 1969 and 1973, respectively. From 1973 to 1974, he was at Toosi University of Technology, Tehran, Iran. From 1974 to 1985 , he was with the Communication Division, Iran Electronics Industries, Shiraz, Iran. In 1985, he joined the Department of Electrical Engineering, Iran University of Science and Technology, Tehran, Iran, where he is a Full Professor of electromagnetic engineering. He has authored and translated several textbooks in Farsi. He has authored or coauthored over 300 papers in international journals and conference. In 2006, he was elected an exemplary nation wide university professor in Iran. $\mathrm{He}$ is an Invited Professor of the Electrical Engineering Group, Academy of Sciences of Iran, and is listed as an elite engineer by the Iranology Foundation. 